Methods A review of the available literature related to application of Octaplas in children.

Results The use of Octaplas in severe patients reduces the possibility of immune and anaphylactic reactions and transfusion - induced acute lung injury. Also, a smaller volume of Octaplas compared to FFP is required to correct coagulopathy. No additional bleeding with reduction in the number of transfusions in liver transplant patients was observed. An increase in platelets has been demonstrated in patients with thrombotic thrombocytopenic purpura who were refractory to FFP. The survival rate in Pediatric Intensive Care Unit (PICU) was higher in children treated with Octaplas compared to FFP. Recent research has shown that in children who received Octaplas during cardiac surgery ACT values were lower with lower doses of heparin, and platelets, APTV, INR, fibrinogen values were higher compared to children who received FFP. Reduction in postoperative infections and shorter stay in PICU were observed. The latest prospective, multicenter study in the United States examined the safety, tolerability, and efficacy of Octaplas in the treatment of children who required replacement of multiple coagulation factors due to cardiac surgery, transplantation and/or liver dysfunction, with coagulopathy and sepsis - related coagulopathy as well as hypoxic encephalopathy. No thromboembolic events associated with hyper fibrinolysis or treatment have been reported. The overall safety, tolerability and efficiency was defined as excellent. Hemostatic parameters measured with INR, PT, APTV, thromboelastography, or thromboelastometry were within the expected range.

Conclusion The results of previous research support the use of Octaplas in children. Octaplas has an advantage over FFP due to its strong hemostatic effect, less frequent side effects, safety and in critically ill children may be associated with improved survival.

\section{RETROSPECTIVE STUDY OF THE USAGE OF CENTRAL VENOUS CATHETERS IN TWO-YEAR PERIOD AT THE DEPARTMENT OF PEDIATRICS IN UNIVERSITY HOSPITAL CENTRE ZAGREB,CROATIA}

Kristina Kljaić ${ }^{\star}$, Nives Živković, Dorotea Šijak, Jurica Vuković. KBC Zagreb

\subsection{6/archdischild-2021-europaediatrics.355}

Aim To analyze methods of central venous catheter (CVC) usage, to establish the state of current practice and perhaps change the approach depending on the analysis of our results. Methods A retrospective study which included a cohort of patients treated at the Department of Pediatrics UHC Zagreb, to whom in the period from January 1th 2018 till December 31th 2019 the CVC was extracted. A sample was formed based on results of microbiological analysis of the CVC tip. The main source of data was the hospital's information system, which was analyzed using descriptive statistics methods. According to the variety of the underlying disease, patients have been divided into 4 groups: congenital anomalies, cardiovascular, hemato - oncological diseases and others.

Results 11,648 children were hospitalized in our Department during a two-year period, when CVC was extracted in 505 $(4.3 \%)$ of all hospitalized children. One CVC had 385, two 81 , three or more 39 of 505 children. A total of 693 catheters were extracted: 449 Broviac, 195 PICC, 38 umbilical, 7 Port-a-Cath and 2 Hickman catheters. The distribution of
CVC by groups was: heart diseases (287), congenital anomalies (224), hemato - oncological (78), other diseases (104). Based on the available data, we singled out the causes of extraction in 91 respondents (i.e. 122 removed catheters). We list them in order: end of treatment (75/122), dysfunction (9/ 122), displacement (6/122), mechanical damage (5/122) and catheter sepsis (2/122). A total of 667 catheters were microbiologically analyzed, 172 of them were positive (25.78\%). The most common agents were: Coagulase - negative Staphylococcus (51), Staphylococcus epidermidis (44) and Candida yeasts (18). There was no difference in the incidence of positive catheters in groups of respondents with different numbers of catheters (I 24.5\%, II 27.5\%, III 27.1\%). Patients with congenital anomalies had a slightly higher incidence of microbiologically positive catheters (32\%), while in cardiovascular, hemato - oncological and other diseases, incidence was almost equal $(22 \%, 25 \%, 26 \%)$.

Conclusion CVC is rarely required in the treatment of our patients, but unavoidable in cardio-surgical, hemato - oncological patients and in many others with complex congenital anomalies. A significant number of catheters had been colonized over the time, but even in the case of an invasive disease, antimicrobial treatment was continued using the same catheter. Its extraction was needed extremely rare. Regardless to the nature of diseases in which the CVC was used, our results suggest the requisite to revise their usage.

\section{Paediatric Nephrology}

\section{OUR EXPERIENCES IN CONTRAST-ENHANCED VOIDING UROSONOGRAPHY IN THE DIAGNOSTICS OF THE VESICOURETERAL REFLUX IN CHILDREN}

Rubelj Karla*, Oletić Lea, Valent Morić Bernardica, Trutin Ivana. Clinical hospital center Sestre Milosrdnice

\subsection{6/archdischild-2021-europaediatrics.356}

Vesicoureteral reflux (VUR) is one of the most common anomalies of the urinary system in children. It is the most common cause of renal scarring and consequently impaired renal function. Contrast-enhanced voiding urosonography (ceVUS) is becoming a recognized method for the diagnosis of VUR in children in specialized centers. The aim of this study is to examine the indications for ceVUS in children, determine the degrees of VUR and their associated characteristics, and possibly predict which children are at higher risk of VUR.

Patient data was collected from medical records of the Institute for Nephrology, Department of Pediatrics of the Sisters of Mercy University Hospital, from September 2016 till December 2018. The following data were taken into consideration: gender, age, characteristics of UTI (relapse, febrility), distribution of pathogens, values of inflammatory parameters (leukocytes and CRP), findings of the urinary tract ultrasound and correlation of these characteristics with the appearance of VUR. Percentages and mean values were used for descriptive statistics purposes. The $\chi 2$ test was used to determine the differences between the two variables and the Student's t-test was used to determine the differences of arithmetic means.

The average age of children was $1.7 \pm 1.1$. Out of 175 children who underwent ceVUS, VUR was detected in 68 (38.9\%), equally for both genders ( $\mathrm{M}$ vs. $\mathrm{F}=32.3 \%: 40.2 \%$;), 
mostly grade II $(41 ; 60.3 \%)$ and grade III $(20 ; 29.4 \%)$. Of the total number of children with a prenatal diagnosis of hydronephrosis, 28,6\% had VUR.

In the group of children with first febrile UTI, VUR was discovered in $30(36,6 \%)$. With regard to VUR, no significant difference was found between normal pathologic ultrasound findings $(38.1 \%$ vs. $41.7 \%$; p 0.739$)$. No difference was found regarding severity of UTI: urine Leukocyte $<20$ vs. urine Leukocyte $>20(40.3 \%: 43.7 \% ; \mathrm{p}=0,787)$, $\mathrm{CRP}<50$ vs. CRP > $50(37.5 \%: 35.5 \% ; \mathrm{p}=0,46)$ as well as between age of children $<1$ year vs. $>1$ year $(34,5 \%: 41,3 \% ; p=0,141$. Significant differences were found between girls and boys $(\mathrm{M}$ vs.F $=19.0 \%$ vs.34.6\%; $\mathrm{p}=0.0466)$.

Analyzed data point to a higher likelihood of background VUR in recurrent UTI; whereas gender, age, urinary tract ultrasound examination, severity, and other causative agents of UTI have no predictive value for VUR detection. ceVUS is one of the best choices in modern nephrology for the detection of VUR with high sensitivity and negligible side effects.

\section{A RARE CASE OF POLYCYSTIC KIDNEY DISEASE AND MULTICYSTIC DYSPLASTIC KIDNEY IN A PEDIATRIC PATIENT}

Kovačević Ana*, Roić Goran, Trutin Ivana, Valent Morić Bernardica. Sestre milosrdnice University Hospital Centre

\subsection{6/archdischild-2021-europaediatrics.357}

Introduction Cystic kidney disease (CyKD) is one of the most important causes of chronic kidney disease (CKD) in children. Multiple kidney cysts can occur unilaterally (e.g. multicystic dysplastic kidney - MCDK) or bilaterally (e.g. autosomal dominant or autosomal recessive polycystic kidney disease) due to genetic or non-genetic (developmental or acquired) disorders. Here we present a rare case of combined polycystic kidney disease (PCKD) and MCDK in a pediatric patient.

Case Report The patient was admitted to our hospital for the first time at the age of two. She was normally developed (weight: $10.8 \mathrm{~kg}-19$ th percentile, height: $90 \mathrm{~cm}-80$ th percentile) with blood pressure (BP) within normal limits.

Estimated glomerular filtration rate (eGFR) at that time was $70.2 \mathrm{~mL} / \mathrm{min}$ with elevated albumin/creatinine ratio 5,2 $\mathrm{mg} / \mathrm{mmol}$ and she was diagnosed with G2A2 stage of CKD. During her prenatal period oligohydramnios and polycystic kidneys were detected. At birth a palpable mass of the left abdomen was found. First sonography after birth showed hydronephrosis of the right kidney and multiple cysts on the left kidney. Micturating cystourethrography was negative for vesicoureteral reflux. Initial magnetic resonance imaging (MRI) showed enlargement of both kidneys with multiple cysts of various sizes on the left kidney and lesser number of diffuse renal cysts on the right kidney. Cysts weren't found neither on the brain nor on the other abdominal organs. Echocardiography was normal. Family history was negative for kidney diseases. The abdominal and kidney ultrasound of both parents and older brother was normal. At the age of two dynamic scintigraphy with Tc-99m revealed nonfunctional left kidney leading to diagnosis of MCDK.
Follow-up ultrasound showed progressive involution of the MCDK left and multiple very small cysts that generate abnormal parenchymal echogenicity (eg, salt-and-pepper sign) on the right kidney. Functional MRI urography showed nonfunctional MCDK left and functional right kidney with lesser number of small cysts. At the age of four arterial hypertension $(130 / 70 \mathrm{mmHg})$ was diagnosed and ACE inhibitor was introduced. There were no signs of liver disease. Abdominal ultrasound revealed no cysts of liver, spleen or pancreas. Gynecological ultrasound and ophtalmological examination were also normal. Due to parental disapproval, genetic testing wasn't performed.

Conclusion We conclude that, although rare, different types of CyKD can be associated and we should consider it when setting the diagnosis. Due to vast differential diagnosis and overlapping clinical presentations of CyKD genetic testing should be performed whenever possible.

\section{RECURRENT MULTI DRUG RESISTANT URINARY TRACT INFECTIONS IN A THREE-YEAR-OLD HOSPITALIZED CHILD WITH HYPOTONIC CEREBRAL PALSY}

Isac Raluca*, A Blescun, Cl Olariu, R Stroescu, M Gafencu, F Horhat, L Chisavu, G Doros. University of medicine and Pharmacy 'Victor Babes' Timisoara, Department of Pediatrics

\subsection{6/archdischild-2021-europaediatrics.358}

Background Catheter associated urinary tract infection (CAUTI) is a common device-acquired infection and represent a potentially harmful reservoir of resistant uropathogens. Guidelines recommend limitation of catheter use, aseptic catheter insertion, sterile equipment, strict hand hygiene, use of smallest catheter possible and maintenance of a closed drainage system.

Klebsiella Pneumoniae is a non-mobile aerobic rod causing a large spectrum of hospital-acquired infections, especially pneumonia or urinary tract infections (UTI), developing intrinsic resistance genes. Treating multi drug resistant(MDR) gram negative pathogens becomes a challenge for the caregiver.

Vesicoureteral reflux(VUR) consists of backflow of urine from the bladder into the ureters. It can be primary or secondary due to abnormal lower urinary tract function and elevated intravesical pressure. Post void residual(PVR) is a hallmark of detrusor underactivity(DUA) in children.

Case Presentation Summary We present the case a three years old boy, hospitalized for viral encephalitis, undergoing artificial respiratory support and urine catheterization for 6 weeks. Neurological status was hypotonic cerebral palsy and secondary urine incontinence in a previously toilet trained child.

First febrile UTI developed two days after removing urine catheter. High resistant Klebsiella pn. (ESBL+, AAC(3)-II) was treated with a ten-day course of Cephtriaxone and Amikacin. Clinical response to treatment was good with sterile urine culture after 96 hours. After treatment, the child had asymptomatic bacteriuria with MDR Klebsiella pn. in spite of rigorous local hygiene, proper hydration and oral Fosfomycin. Second febrile UTI was accompanied by febrile seizures, and urine culture was positive with MDR Klebsiella (+ESBL or +HL AmpC, Carbapenem impermeability). Treatment with high dose Meropenem (40mg/kg/dose) for twelve days was successful. Third febrile UTI occurred five days after finishing treatment with same MDR Klebsiella strain. Once again, fourteenday high dose Meropenem course was successful. Fourth 